\title{
Compressive Sampling of Multiple Sparse Signals Having Common Support Using Finite Rate of Innovation Principles
}

\author{
Ali Hormati, Member, IEEE, and Martin Vetterli, Fellow, IEEE
}

\begin{abstract}
In sensor networks and communication systems, one is often confronted with sampling multiple sparse signals having a common support set. Multipath channels in a multiple-input multiple-output (MIMO) wireless communication setting is an interesting example where one generally needs to perform channel estimation for each transmit-receive antenna pair. MIMO multipath channels are usually (approximately) sparse and satisfy the common-support property whenever the distances between the antennas are small compared to the distance the electromagnetic wave can travel in the time corresponding to the inverse bandwidth of the communication system. This assumption is satisfied by small and medium bandwidth communication systems like OFDM and CDMA.

This leads us to extend the finite rate of innovation sampling and reconstruction scheme to the sparse common-support scenario (SCS-FRI), in which input signals contain Diracs with common locations but arbitrary weights. The goal is to efficiently reconstruct the input signals from a set of uniform samples, making use of the common-support property to improve robustness. We first find the best theoretical performance for the SCS-FRI setup by deriving the Cramér-Rao lower bound. Our results show that for a set of well-separated Diracs, it is the total energy of the Diracs at each common position which determines the bound. We then propose a multichannel reconstruction algorithm and compare its performance with the Cramér-Rao lower bound. Numerical results clearly demonstrate the effectiveness of our proposed sampling and reconstruction scheme in low SNR regimes.
\end{abstract}

Index Terms-Annihilating filter, compressed sensing, Cramér-Rao bound, finite rate of innovation, MIMO channel estimation, multichannel sampling.

\section{INTRODUCTION}

C ONSIDER a set of continuous-time sparse signals, each composed of $K$ Diracs, which are sampled by a set of independent sampling devices and then reconstructed jointly. If the signals are totally independent, independent sensing and recovery scheme would be optimal. However, in the presence

Manuscript received February 04, 2011; revised March 14, 2011; accepted March 16, 2011. Date of publication March 24, 2011; date of current version March 31, 2011. This work was supported by Qualcomm, Inc, ERC Grant 247006, and SNSF Grant 200021-121935. The associate editor coordinating the review of this manuscript and approving it for publication was Dr. Jared W. Tanner.

A. Hormati is with the School of Computer and Communication Sciences, Ecole Polytechnique Fédérale de Lausanne (EPFL), CH-1015 Lausanne, Switzerland (e-mail: ali.hormati@epfl.ch).

M. Vetterli is with the School of Computer and Communication Sciences, Ecole Polytechnique Fédérale de Lausanne (EPFL), CH-1015 Lausanne, Switzerland, and also with the Department of Electrical Engineering and Computer Sciences, University of California, Berkeley, CA 94720 USA (e-mail: martin.vetterli@epfl.ch).

Digital Object Identifier 10.1109/LSP.2011.2131649

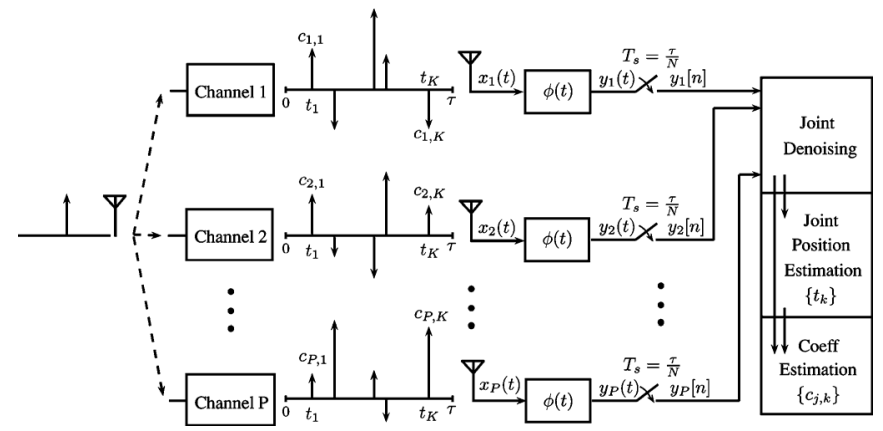

Fig. 1. SCS-FRI sampling and reconstruction scheme in a multi-antenna channel estimation setting with $P$ receiving antennas.

of some common information, a carefully designed sampling and reconstruction procedure would be able to reduce the total number of measurements and/or make it more robust to the uncertainties in the system.

Classical finite rate of innovation (FRI) sampling scheme provides efficient and robust solutions to sample and reconstruct special classes of continuous-time signals having finite degrees of freedom per unit of time, such as, for example, stream of Diracs or piecewise polynomials [1]. In this paper, we extend the FRI sampling and reconstruction technique to a sparse common-support setup (SCS-FRI). In this model [2], the sparse signals, each consisting of $K$ continuous-time Diracs, share common nonzero locations but can have arbitrary weights. We propose special distributed sensing and joint recovery mechanisms which employ the common location property to efficiently estimate the positions and the weights of the Diracs in the input signals. More specifically, in the SCS-FRI setup, each signal is first passed through a lowpass sampling kernel and is then sampled uniformly. Then, all the samples are given to a joint recovery algorithm in order to estimate the positions and weights of the Diracs (see Fig. 1).

An interesting application well-modeled by SCS-FRI is the pilot-assisted multipath channel estimation in a radio-frequency multi-antenna communication system. In such systems, pilot signals are sent by the transmitter to the multi-antenna receiver in specific arrangements to estimate the underlying time-varying fading channels. The multipath channels seen by the set of nearby receiving antennas share the same nonzero positions (corresponding to the paths) with different weights resulting from different fading and shadowing effects. Therefore, using our joint SCS-FRI recovery method proposed in this paper, one can estimate the multipath channels in a robust and efficient way.

After a precise definition of the model in Section II, we state in Section III our joint reconstruction algorithm for the 
SCS-FRI problem. Then, in Section IV we find the best theoretical performance in the SCS-FRI setting by deriving the Cramér-Rao lower bound (CRB). In the case of total constant energy, the derived bound is invariant to the change in the weights of the Diracs; This issue is discussed separately in Section V. Finally, we run a set of numerical experiments in Section VI which shows that the proposed recovery algorithm is effective in achieving the CRB in low SNR; a regime which is of interest in wireless multi-antenna communication systems.

\section{Signal Model and Problem Statement}

Consider a set of $P \tau$-periodic FRI signals consisting of $K$ Diracs with a common support set

$$
x_{j}(t)=\sum_{k=1}^{K} c_{j, k} \delta\left(t-t_{k}\right) \quad j=1, \ldots, P
$$

where $c_{j, k}$ is the weight of the $k$ th Dirac of the signal number $j$ and $\left\{t_{k}\right\}_{k=1}^{K}$ denotes the set of common positions in the range $[0, \tau[$. For the sampling part, each signal is independently passed through a lowpass sampling kernel $\phi(t)$ given by

$$
\phi(t)=\operatorname{sinc}(B t) \quad B=\frac{2 L+1}{\tau}, \quad L \geq K .
$$

The output of the sampling kernel is then uniformly sampled at the rate of $f_{s}=\left(1 / T_{s}\right)=N / \tau$ samples per second with $N \geq B \tau$. The samples are given by

$$
y_{j}[n]=\sum_{k=1}^{K} c_{j, k} \varphi\left(n T_{s}-t_{k}\right)+\epsilon_{j, n} \quad n=0, \ldots, N-1
$$

where $\varphi(t)$ is the $\tau$-periodic sinc function or Dirichlet kernel:

$$
\varphi(t)=\sum_{k \in \mathbb{Z}} \operatorname{sinc}(B(t-k \tau))=\frac{\sin (\pi B t)}{B \tau \sin \left(\frac{\pi t}{\tau}\right)} .
$$

Given the set of samples $\boldsymbol{y}_{j}, j=1, \ldots, P$, the goal is to efficiently estimate the set of common positions $\left\{t_{k}\right\}_{k=1}^{K}$ and weights $\left\{c_{j, k}\right\}$ of the Diracs. Note that using the sampling parameters mentioned above, it is possible to compute the $2 L+1$ consecutive lowpass Fourier series coefficients of each channel from the samples. Estimating these coefficients is the first step in the reconstruction phase as detailed in the next section.

\section{SCS-FRI RECONSTRUCTION}

The reconstruction algorithm in the classic FRI setting is based on the fact that the Fourier series coefficients of the input signal is composed of sum of exponentials and therefore, techniques well-known in the field of spectral estimation, such as the annihilating filter method, can be used to find the amplitudes and frequencies of the underlying complex exponentials. The frequencies will give the locations of the Diracs while their weights are computable from the amplitudes. In a noisy setting, the parametric estimation procedure can be made robust by using the total least-squares approximation, possibly enhanced by an initial denoising by using an iterative denoising algorithm due to Cadzow [3], [4]. In the SCS-FRI reconstruction, because of the common support property, one needs to find only one annihilating filter common to all the channels. We adapt the Cadzow denoising procedure in the single-channel FRI reconstruction scheme to incorporate the common support property of the set of sparse signals which makes the position estimations much more robust to the noise in the samples.

Taking the Fourier transform of the samples of each signal, we get

$$
Y_{j}[m]=\sum_{k=1}^{K} c_{j, k} \omega^{m t_{k}}+\varepsilon_{j, m} \quad m=-L, \ldots, L
$$

where $\omega=e^{-i 2 \pi / \tau}, c_{j, k}$ denotes the $k$ th nonzero coefficient of the signal $j$ and $\varepsilon_{j, m}$ is the additive noise. The key step in the SCS-FRI reconstruction is the following joint iterative denoising procedure which includes the samples from all the channels simultaneously. We first build the composite matrix $\boldsymbol{H}$ from the set of matrices $\left\{\boldsymbol{H}_{j}\right\}_{j=1}^{P}$ of size $(L+1) \times(L+1)$ as

$$
\boldsymbol{H}=\left[\begin{array}{c}
\boldsymbol{H}_{1} \\
\boldsymbol{H}_{2} \\
\vdots \\
\boldsymbol{H}_{P}
\end{array}\right] \quad \text { where } \boldsymbol{H}_{j}(i, k)=Y_{j}[i-k] .
$$

In the noiseless case, matrix $\boldsymbol{H}$ has two properties.

1) It has the block Toeplitz structure, i.e., all submatrices $\left\{\boldsymbol{H}_{j}\right\}_{j=1}^{P}$ are Toeplitz.

2) It is of rank $K$.

In the noisy case, these two properties are not initially satisfied simultaneously, but can be subsequently enforced by alternatively performing the following two steps.

1) Enforce rank $K$ by setting the $L-K+1$ smallest singular values of $\boldsymbol{H}$ to zero.

2) Enforce the block Toeplitz structure by averaging the coefficients along the diagonals of each submatrix $\boldsymbol{H}_{j}$.

The above procedure is guaranteed to converge to a matrix which exhibits the desired properties [3]. The iterations stop whenever the ratio of the $(K+1)$ th singular value to the $K$ th one, $\sigma_{K+1} / \sigma_{K}$, falls below a predetermined threshold. The denoised DFT coefficients are then extracted from the first row and first column of each submatrix $\boldsymbol{H}_{j}$. The denoised coefficients are then used to build a set of matrices $\left\{\boldsymbol{G}_{j}\right\}_{j=1}^{P}$ of size $(2 L-K+1) \times(K+1)$ of the form (3). The annihilating filter $A(z)$ is a polynomial of degree $K$ of the form

$$
\begin{aligned}
A(z) & =\prod_{k=1}^{K}\left(1-e^{-i 2 \pi t_{k} / \tau} z^{-1}\right) \\
& =1+A[1] z^{-1}+\cdots+A[K] z^{-K} .
\end{aligned}
$$

The annihilating property of $A(z)$ is denoted as

$$
\left(A * \boldsymbol{Y}_{j}\right)[m]=\sum_{k=0}^{K} A[k] Y_{j}[m-k]=0 \quad j=1, \ldots, P .
$$

The coefficients of the annihilating filter $A(z)$ are found such that they satisfy

$$
\left[\begin{array}{c}
\boldsymbol{G}_{1} \\
\boldsymbol{G}_{2} \\
\vdots \\
\boldsymbol{G}_{P}
\end{array}\right]\left[\begin{array}{c}
A[0] \\
A[1] \\
\vdots \\
A[K]
\end{array}\right]=0 .
$$

This system of equations is solved for the annihilating filter coefficients. The roots of the annihilating filter provide us with the positions of the nonzero entries. Finally, the weights of the Diracs are calculated from the denoised measurements by solving the linear system of (2). 


\section{SCS-FRI CRAMÉR-RAO BOUND}

To assess the performance of the proposed reconstruction algorithm for the SCS-FRI scenario, it is essential to find theoretical limits on the performance of any reconstruction algorithm. For this purpose, the Cramér-Rao bound [5], [6] provides a lower bound on the covariance matrix of any unbiased algorithm which estimates the innovations of the FRI signals.

In [4], the authors derive the Cramér-Rao lower bound for estimating the positions and weights of the Diracs in a singlechannel FRI setup. Considering a single Dirac, the minimal relative uncertainties on the location of the Dirac, $t_{1}$, and on its weight, $c_{1}$, are given by

$$
\begin{aligned}
& \mathbb{E}\left[\left(\frac{\triangle t_{1}}{\tau}\right)^{2}\right] \geq \frac{3(2 L+1)}{4 \pi^{2} N L(L+1)} \text { PSNR }^{-1}, \\
& \mathbb{E}\left[\left(\frac{\triangle c_{1}}{c_{1}}\right)^{2}\right] \geq \frac{2 L+1}{N} \text { PSNR }^{-1}
\end{aligned}
$$

where PSNR $=c_{1}^{2} / \sigma^{2}$ is the input peak signal to noise ratio. In the case of multiple Diracs, the Cramér-Rao formula for one Dirac still holds approximately when the distances between Diracs are more than $2 / B$.

Although it is possible to derive the CRB for the general case, for the sake of avoiding unnecessary complex formulas, we calculate the CRB for $P$ signals having one Dirac at the same position. As shown below in Theorem 1, the results indicate that in this setup, it is the total energy of the Diracs that determines the minimum uncertainty in the estimation of the unknown position.

Consider the noisy samples of the signal $j$ at the output of the lowpass sampling kernel given by

$$
y_{j}[n]=c_{j} \varphi\left(n T_{s}-t_{1}\right)+\varepsilon_{j, n} \quad n=0, \ldots, N-1
$$

where $c_{j}$ is the weight, $T_{s}=\tau / N, \varepsilon_{j, n}$ is a zero mean white Gaussian noise with variance $\sigma^{2}$ and $\varphi(t)$ is the Dirichlet kernel (1) with bandwidth parameter $B=(2 L+1) / \tau$.

Theorem 1: The minimal uncertainties on the estimation of the position $t_{1}$ and weights $\left\{c_{j}\right\}_{j=1}^{P}$ in a single-Dirac SCS-FRI scenario with $P$ signals are given by

$$
\begin{aligned}
& \mathbb{E}\left[\left(\frac{\triangle t_{1}}{\tau}\right)^{2}\right] \geq \frac{3(2 L+1)}{4 \pi^{2} N L(L+1)} \text { ESNR }^{-1} \\
& \mathbb{E}\left[\left(\frac{\triangle c_{j}}{c_{j}}\right)^{2}\right] \geq \frac{2 L+1}{N} \text { PSNR }_{j}^{-1} \quad j=1, \ldots, P
\end{aligned}
$$

where ESNR $=\left(1 / \sigma^{2}\right) \sum_{j=1}^{P} c_{j}^{2}$ denotes the effective signal to noise ratio and $\mathrm{PSNR}_{j}=c_{j}^{2} / \sigma^{2}$.

Proof: We build a vector $\boldsymbol{y}$ of size $N \times P$ composed of all the samples from the $P$ signals. Since the additive noise is i.i.d. Gaussian, $\boldsymbol{y}$ has a multivariate Gaussian distribution $f_{\boldsymbol{\theta}}(\boldsymbol{y})$ with a diagonal covariance matrix. Setting the vector of parameters as $\boldsymbol{\theta}=\left(t_{1}, c_{1}, c_{2}, \ldots, c_{P}\right)$, we compute the diagonal components of the Fisher information matrix as

$$
\begin{aligned}
\boldsymbol{J}_{1,1} & =\mathbb{E}\left[\left(\frac{\partial}{\partial t_{1}} \ln f_{\boldsymbol{\theta}}(\boldsymbol{y})\right)^{2}\right] \\
& =\frac{\left(\sum_{\ell=1}^{P} c_{\ell}^{2}\right) 4 \pi^{2} N M(M+1)}{3(2 M+1) \tau^{2} \sigma^{2}}
\end{aligned}
$$

$$
\boldsymbol{J}_{\ell+1, \ell+1}=\mathbb{E}\left[\left(\frac{\partial}{\partial c_{\ell}} \ln f_{\boldsymbol{\theta}}(\boldsymbol{y})\right)^{2}\right]=\frac{N}{(2 M+1) \sigma^{2}}
$$

for $\ell=1, \ldots, P$. The off diagonal terms are computed in the same way as

$$
\begin{aligned}
\boldsymbol{J}_{\ell+1, \ell^{\prime}+1} & =\mathbb{E}\left[\frac{\partial}{\partial c_{\ell}} \ln f_{\boldsymbol{\theta}}(\boldsymbol{y}) \frac{\partial}{\partial c_{\ell^{\prime}}} \ln f_{\boldsymbol{\theta}}(\boldsymbol{y})\right] \\
& =0 \quad \ell, \ell^{\prime}=1, \ldots, P \\
\boldsymbol{J}_{1, \ell+1} & =\mathbb{E}\left[\frac{\partial}{\partial t_{1}} \ln f_{\boldsymbol{\theta}}(\boldsymbol{y}) \frac{\partial}{\partial c_{\ell}} \ln f_{\boldsymbol{\theta}}(\boldsymbol{y})\right] \\
& =0 \quad \ell=1, \ldots, P .
\end{aligned}
$$

The proof follows by inverting the diagonal Fisher information matrix.

Comparing the CRB for the single-channel and SCS-FRI settings, we conclude that in the latter, it is the sum of the energy of the Diracs that determines the uncertainty of the position estimation. However, the uncertainty in recovering the weight of the Dirac in each channel depends only on the corresponding Dirac weight.

\section{POWER Distribution}

Consider a single-Dirac SCS-FRI scenario with $P$ signals and let $\left\{c_{j}\right\}_{j=1}^{P}$ and $t_{1}$ represent the weights and the common location of the Diracs, respectively. We study the following two scenarios.

1) The recovery algorithm estimates the position of the Dirac in each signal separately using the single-channel FRI reconstruction algorithm and averages the results.

2) The recovery algorithm estimates the common location of the Diracs using the joint reconstruction technique of Section III.

We want to investigate the effect of the change in the power distribution among the channels on the minimum uncertainties that can be achieved in the two scenarios mentioned above.

Theorem 2: Assume that the recovery algorithms are unbiased and achieve the Cramér-Rao lower bound for both the single-channel and multichannel settings. Let $\Gamma(\mathrm{I})$ and $\Gamma(\mathrm{II}) \mathrm{de}-$ note the uncertainties in position estimation for the scenarios I and II, respectively. Then, we have $\Gamma(\mathrm{II}) \leq \Gamma(\mathrm{I})$ with equality if and only if the total power is distributed uniformly among the channels.

Proof: Let $\triangle t_{i}, i=1, \ldots, P$ and $\triangle t_{\mathrm{M}}$ denote the estimation errors of the single-channel and multichannel recovery algorithms, respectively. Let $\alpha=\left(3 \sigma^{2}(2 L+1) / 4 \pi^{2} N L(L+1)\right)$. From Theorem 1 we have

$$
\Gamma(\mathrm{II})=\mathbb{E}\left[\left(\frac{\triangle t_{\mathrm{M}}}{\tau}\right)^{2}\right]=\frac{\alpha}{\sum_{i=1}^{P} c_{i}^{2}} .
$$

We write

$$
\begin{aligned}
\Gamma(\mathrm{I}) & =\mathbb{E}\left[\left(\frac{\frac{1}{P} \sum_{i=1}^{P} \Delta t_{i}}{\tau}\right)^{2}\right] \stackrel{(a)}{=} \frac{1}{P^{2}} \sum_{i=1}^{P} \mathbb{E}\left[\left(\frac{\triangle t_{i}}{\tau}\right)^{2}\right] \\
\stackrel{(b)}{=} & \frac{1}{P^{2}} \sum_{i=1}^{P} \frac{\alpha}{c_{i}^{2}}
\end{aligned}
$$


where in $(a)$ we used the fact that the estimates are all unbiased and independent and in (b), we used (4). To get the final result, we write

$$
\left(\sum_{i=1}^{P} c_{i}^{2}\right)\left(\sum_{i=1}^{P} \frac{1}{c_{i}^{2}}\right)=P+\sum_{i=1}^{P} \sum_{j \neq i}^{P} \frac{c_{i}^{2}}{c_{j}^{2}} \stackrel{(a)}{\geq} P^{2}
$$

where in $(a)$ we used the inequality $x+1 / x \geq 2$. Combining (5), (6) and (7), we get $\Gamma(\mathrm{II}) \leq \Gamma(\mathrm{I})$. Note that in (7), the equality is achieved if and only if $c_{1}^{2}=c_{2}^{2}=\ldots=c_{P}^{2}$.

As pointed out in [4], the single-channel FRI reconstruction algorithm achieves the CRB above an SNR threshold that depends on the parameters of the sampling system. As is shown using numerical simulations in Section VI, this SNR threshold to achieve the Cramér-Rao lower bound decreases by using the SCS-FRI recovery algorithm. Achieving the CRB and being unbiased, the result of Theorem 2 indicates that whenever there is an unequal power distribution between the channels, the uncertainty of the location estimation is reduced by using the multichannel reconstruction algorithm compared to the strategy that makes the average of the single-channel reconstructions.

\section{Simulation Results}

In the SCS-FRI setup, we consider a set of $P=6$ periodic signals each containing a single Dirac. The Diracs share the same location $t_{1}$. We take $N=2 L+1$ samples from each signal with $L$ in the set $\{2,5,10\}$. Independent and identically distributed Gaussian noise of variance $\sigma^{2}$ is added to the measurements to get SCS-SNR from $-10 \mathrm{~dB}$ to $40 \mathrm{~dB}$. We define SCS-SNR as the ratio between the total signal power and the total noise power

$$
\begin{aligned}
\mathrm{SCS}-\mathrm{SNR} & =\frac{\sum_{j=1}^{P} \sum_{n=0}^{N-1} c_{j}^{2} \varphi^{2}\left(n T_{s}-t_{1}\right)}{N P \sigma^{2}} \\
& =\frac{\frac{1}{P} \sum_{j=1}^{P} c_{j}^{2}}{\sigma^{2}(2 L+1)}
\end{aligned}
$$

where $\left\{c_{j}\right\}_{j=1}^{P}$ denotes the weights of the Diracs. In the first set of simulations, the weights of the Diracs are equal. We average the uncertainty on the estimation of the location $t_{1}$ for the SCS-FRI reconstruction scheme and the single-channel+averaging scheme (see Section V) over 10000 realizations of the noise. The results are shown in Fig. 2(a). As shown in Theorem 2 for the even distribution of power, the performance of the two algorithms are the same in the high SNR regime when all the single-channel uncertainties approach the CRB. However, compared to the single-channel and averaging scheme, the SCS-FRI reconstruction scheme approaches the CRB at a lower threshold SNR. This can be explained by the fact that SCS-FRI first estimates the annihilating filter coefficients using the data from all the channels and then finds the roots, whereas the traditional method first finds the roots and then makes the average. In Fig. 2(b), we consider the parameters $P=6$ and $L=5$ in an unequal power distribution setting given by the power ratios $(1$, $2,3,4,5,6)$. We can see that the performance of the SCS-FRI reconstruction scheme is not affected by the uneven power distribution among the signals, while the performance of the averaging scheme deteriorates considerably. Although not shown here, the proposed reconstruction algorithm behaves similarly in the case of multiple Diracs separated by more than $2 / B$.

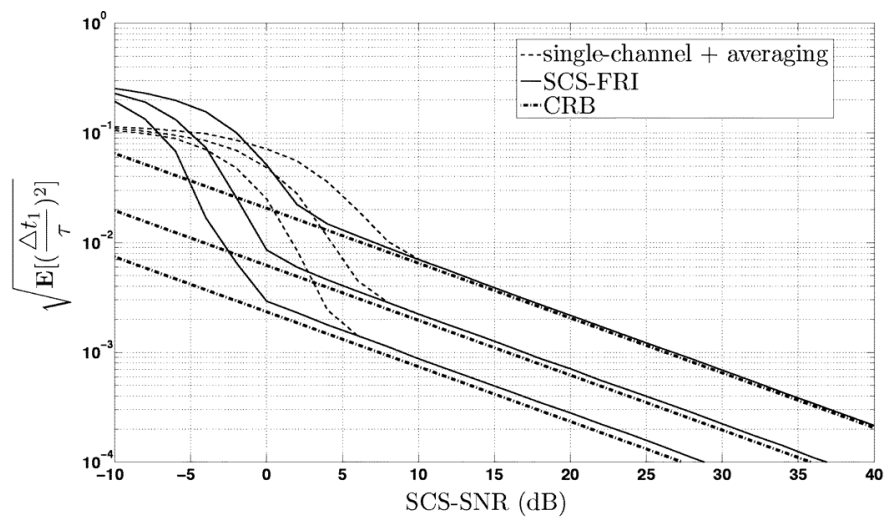

(a)

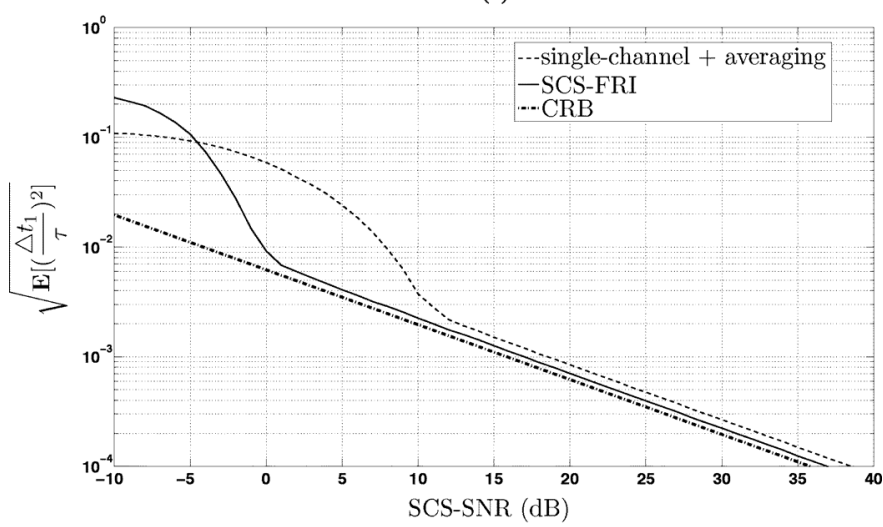

(b)

Fig. 2. Performances of the SCS-FRI reconstruction, the single-channel + averaging reconstruction along with the Cramér-Rao lower bound. (a) Six equalpower signals with 5,11 and 21 samples per signal (from top to bottom). (b) Six signals with power distribution ratios $(1,2,3,4,5,6)$ and 11 samples per signal.

\section{CONCLUSION}

We investigated the SCS-FRI scenario in which the goal is to design efficient techniques to sample and reconstruct a set of FRI signals having common-support property. We proposed special sampling and reconstruction techniques for the SCS-FRI model. By deriving the Cramér-Rao lower bound and using numerical simulations, we showed that the proposed SCS-FRI reconstruction algorithm achieves the bound starting from lower SNR compared to the classical single-channel FRI reconstruction techniques. Our method has a promising application in improving the channel estimation quality for multi-antenna communication systems.

\section{REFERENCES}

[1] M. Vetterli, P. Marziliano, and T. Blu, "Sampling signals with finite rate of innovation," IEEE Trans. Signal Process., vol. 50, no. 6, pp. $1417-1428$, Jun. 2002.

[2] M. F. Duarte, S. Sarvotham, D. Baron, M. B. Wakin, and R. G. Baraniuk, "Distributed compressed sensing of jointly sparse signals," in Asilomar Conf. Signals, Systems and Computers, Oct. 2005, vol. 24, pp. $1537-1541$.

[3] J. A. Cadzow, "Signal enhancement-A composite property mapping algorithm," IEEE Trans. Acoust., Speech, Signal Process., vol. 36, no. 1, pp. 49-67, Jan. 1988

[4] T. Blu, P. L. Dragotti, M. Vetterli, P. Marziliano, and L. Coulot, "Sparse sampling of signal innovations," IEEE Signal Process. Mag., vol. 25, no. 2, pp. 31-40, 2008.

[5] H. Cramér, Mathematical Methods of Statistics. Princeton, NJ: Princeton Univ. Press, 1946.

[6] C. R. Rao, "Information and the accuracy attainable in the estimation of statistical parameters," Bull. Calcutta Math. Soc., vol. 37, pp. 81-89, 1945. 